\title{
Magnetism, the Unknown Side of Health
}

\author{
Anna CM Backerra* \\ Institute of Theoretical and Applied Micro Magnetism, The Netherlands \\ *Corresponding Author: Anna CM Backerra, E-mail: anna.backerra@ \\ itammagnetics.com
}

Received: October 31, 2021

Published: December 20, 2021

C All rights are reserved by Anna CM

Backerra.

\begin{abstract}
Twin physics is a theoretical tool to describe phenomena in a complementary way, based upon results of quantum mechanics, Heisenberg's uncertainty principle and Einstein's relativity theory. According to this theory, four distinct types of electrons are described. Two of these types are surrounded by a finite magnetic space, having an estimated size of one order larger than that of middle-sized molecules. The magnetic space is not fully determined by the movement of the belonging electron, but has a certain amount of freedom in strength and direction of the magnetic field inside. This gives it the ability to adapt to, and connect with the magnetic spaces of protons and neutrons, forming a magnetic cell around a molecule. To be able to investigate the influence of magnetic cells on human health in general, twin physics is transformed into a simplified version called the twin model. Possible consequences of the specific features of magnetic cells for human health are discussed, with special attention for the corona crisis.
\end{abstract}

Keywords: Twin Physics; Complementarity; Magnetic Cell; Magnetic Presence; Corona; Mental Health; Pharmaceutics

\section{Introduction}

There is an intimate historical connection between scientific inventions and health care, as appears from many examples of which we will mention only a few. Optical lenses developed in physics were used to compensate a poor vision; later the single lens was developed into a comfortable pair of glasses and in the $20^{\text {th }}$ century the chemical invention to produce plastic from petroleum made it possible to wear lenses that were invisible. The invention of $x$-rays at the end of the $19^{\text {th }}$ century, by which bones inside a body became visible, started as a fairground attraction but soon was developed into a tool for examining broken bones; later a better control over the frequencies of radiation resulted in a therapy to treat cancer. Exactly 200 years ago Faraday invented the electric engine and the dynamo, and in 1879 the light bulb was invented by Edison; nowadays health care without electricity is almost unthinkable.
Although we know that electricity is inseparably connected to magnetism, expressed by the laws of Maxwell, the understanding of magnetism stays remarkably far behind. The origin of this difference can be found in two details of these laws. First, a magnetic monopole is lacking, so magnetism cannot be controlled as easily as electric charge. Second, at the time physics was supposed to be identical with the describing mathematics, based upon an infinite coordinate system, and so magnetic fields still are supposed to add up like an elusive cloud, gradually fading out to infinity.

In a theoretical model called twin physics, developed since 2004 and published since 2010 [1], these two problems are already solved from the beginning. This was not done to discover more about magnetics, but to explain a quantum mechanical experiment in 1927 [2] and to fill the gap between quantum mechanics and 
relativity theory. The first step was simply abandoning the classical supposition that space is infinite. The second step was embracing the conviction of Heisenberg [3] that all phenomena in the universe can be described in a complementary way. The third step was adopting the mathematical definition of complementarity by Jammer [4] in a practical way.

With the obtained theory it was possible to describe four distinct types of electrons [5,6], two of which existing inside a finite magnetic field, which we call a magnetic space. This opened the possibility to investigate magnetism in an entirely new way.

In fact, the necessity to develop more insight in magnetism is becoming more important. The use of magnetism as a therapeutic tool has been known for a long time, but without a scientific basis. In the last few years the number of people complaining about the influence of electromagnetic smog is growing. In the previous paper we explained that the corona virus according to twin physics has a relative extensive magnetic field around it, adding readily to its contagiousness. In general, medicines are composed without being aware of the possible influence of their magnetic features.

This paper is a sequel to a publication in July 2020 [7] about the corona virus. Here we will discuss the results of twin physics considering human health more in general.

\section{About Twin Physics}

Twin physics is a theoretical model describing physical phenomena in a complementary way, based upon results of quantum mechanics, the Heisenberg's uncertainty principle and Einstein's relativity theory. Most characteristic is, that space is supposed to consist of overlapping finite, energetic objects, being as important as mass. An elementary mass is an object with a very high energy density; an elementary space is an object with a very low energy density but not zero. To construct twin physics, we used the definition of complementarity by Jammer [4], stating that two descriptions A and B are complementary if they are distinct, refer to the same subject, exclude each other and each on its own cannot describe the subject completely [8].

Twin physics provides a phenomenological view on physics. Phenomena are described in a geometric way, allowing to incorporate uncertainty, instead of searching for algebraic details of increasing complexity and accuracy. The explicit transformation of mathematics into physical space and time by a transformation operator, removing items which do not exist in the physical reality, makes it possible to describe the hypothetical magnetic monopole. In this way we can deal with electromagnetic features of particles and spaces much easier than in the classical way. A spherical coordinate system is used, so that particles may be revolving around a central point without using the concept of a force. All details about basic phenomena are collected according to set theory [9], a mathematical technique allowing to combine items of different dimensions, like time and space. This approach makes it relatively easy to apply twin physics to specific experimental problems without losing the overview.

In the first publication of 2010 [1], the starting points are explained, expressed in a complementary mathematical system. In later publications this is applied to several well-known areas of physics to see if it works, and after that we applied it to actual physical problems. The obtained results gave us enough confidence to continue with the application to human health; we will mention some of them below.

Using twin physics, the laws of Maxwell are deduced and understood in an extremely concise and clear way, without introducing them in advance, by inserting an arbitrary radial and an arbitrary circular field in the same formulation as the one we used for space and time [10].

A straightforward relation between two constants of nature, namely the velocity of light (concerning phenomena at the largest scale), and Planck's constant (concerning phenomena at the smallest scale) is deduced [11].

Four types of electrons are described [5,6], two of which exist inside a magnetic space, being valence- and conduction-electrons. This explains why graphene has a very high conductivity in comparison with other forms of carbon; it opens new ways to describe nano materials. The other two types occur in protium and in the lowest level of molecules, respectively. Also two types of protons and three types of neutrons are found [5], occurring in regular molecules and also existing inside a magnetic space. These magnetic spaces add up to a magnetic cell around the molecule.

Recently we managed to describe in detail how a photon is absorbed by an electron, bound to a proton, in which the magnetic 
cell plays the main role. This was the last step which convinced us that magnetic cells actually do exist. This is presented at the conference "Laser, Optics and Photonics", Brussels, September 13-14, 2021, to be published in Nano Progress.

The magnetic space of an electron, a proton or a neutron may be spherical or an intersection of spheres, and its strength may be reinforced by magnetic spaces of elementary particles nearby. The most astonishing feature of a magnetic space is its uniqueness: two identical magnetic spaces cannot exist. Even though it is restricted by the laws of Maxwell, the magnetic field inside is unique in terms of the strength and directions of the magnetic vectors [10]. The scope of this freedom still has to be investigated.

In short, the uniqueness of each magnetic cell is the most interesting aspect of twin physics considering the application on human beings. This is facilitated by a simplified version, called the twin model [7], being more focused on that.

We suppose that this maximum size is at least molecular (a few nanometers, so in the order of $10^{-9}$ to $10^{-8} \mathrm{~m}$ ), but presumably one or two orders of magnitude larger (a few micrometers, so in the order of $10^{-6} \mathrm{~m}$ ).

\section{Discussion}

The human body consists of mass, consisting of a huge number of molecules and together forming a coherent material unity. Each of these molecules is embedded in a magnetic cell; we suppose that in a similar way, all these magnetic cells together form a coherent magnetic presence in and around the body. In daily life, the magnetic presence is called the spirit, serving non-biological functions like religion, music, poetry, painting and other arts. Talents in these subjects cannot be measured in the child, only tried out, which is in agreement with the fact that magnetism cannot be kept under control easily (like a battery for electricity). The brain is electrically the most active part of the body, influencing the magnetic presence by sending small amounts of electricity through the nervous system. The complex of the brain functions will be called the mind, which will be considered separately of the remaining part of the body.

So a human has mass (a body), a magnetic presence (the spirit) and brains (the mind). The functions of the body serve the biological functions of the human being, switching between two phases of the organs and kept under control by the mind. Another functional unity is formed by mind and spirit, the mind acting as an intermediate between body and spirit, and the spirit serving non-biological functions. Thus the mind is the connection between body and spirit.

We suppose that the more people engage in sports and outdoor activities, the more their body (muscles, bones, intestines etc.) will be healthy and strong. Similarly, the more people develop their artistic, musical and religious, in general called spiritual possibilities, the more their magnetic presence will be healthy and strong.

The magnetic presence should not be confused with the electromagnetic field of a network. The difference is that the field of an antenna supports internet connections by receiving and sending information with the velocity of light, whilst, by contrast, a magnetic presence is a local phenomenon, being unique for each human being and solidly connected to it.

This uniqueness is hardly represented in health care, although, according to its physical background, it represents one out the three aspects (body, mind, spirit) of each human being. Pharmaceutical research is directed to influencing the body without involving the unique magnetic presence, and thus according to the twin model, doctors may erroneously suppose that a drug will help each patient in the same way. On the other hand, many alternative therapies use mainly the signals of the magnetic presence, but because there is no scientific validation, therapists are only guided by their intuition and their insights cannot be applied in a broader sense.

Another feature of regular health care is the use of test subjects. It is very likely that people who volunteer for these trials are no artists, musicians, poets and so on, because it is very well known that people with these professions have a higher level of uniqueness than others and so in general they will be more apprehensive about participating.

This suggests that medical and pharmaceutical research not only overlooks the spirit, but that also the possible effects of medication upon spiritually strongly developed people remain under the radar. Thus for this type of people, medicines might worsen diseases instead of curing them. 
In a previous paper [7], we explained that the corona virus, because of its form, is expected to have a relatively large region of magnetic influence around it, and why people having a weak magnetic presence are expected to be more susceptible to it and will suffer more than those having a strong and healthy magnetic presence. In the same paper, we explained that a computer cannot transfer magnetic information and so, during the time that you use the computer, the spirit cannot be involved as an active part in the interaction, which will gradually upset the balance between the mind and the spirit.

It's about time to explore the magnetic presence of people at the largest level, not only to supply in health care for artists, but basically to raise the health level for the population at large. All individuals possess a unique magnetic presence and will experience periods of poor health; at those instances, the work of artists (i.e. persons with well-developed magnetic presence) in whatever form could help, including giving workshops and lessons.

New research is always difficult because it is hard to know where to begin and what to expect. The easiest way to find out if there is a possible relation between infection with the corona virus and the condition of the magnetic presence seems to be to study professional musicians in orchestras, finding out how many of them had to go to a hospital because of the infection with covid, and how many of them needed intensive care.

Comparing this with a similar group of people working together and having non-artistic professions, like nurses in a hospital, could give a first indication. A second way could be to make a similar inventory of people, working as a certified artist, compared with other people working alone in a non-artistic profession like accountants. A third way would be to compare the percentage of artists and musicians refusing the vaccination with a control group.

In most countries the governments try to convince as many people as possible to get vaccinated against covid. It is an open question if one hundred percent degree of vaccination indeed would improve the health of the population in the future. If the different situation for musicians and artists is not respected by forcing them to accept vaccination, this might result in a relevant weakening which could be dangerous in time. If a variant of the virus would pop up, and if indeed a stronger artistic education would reinforce the average resilience, then the people who could carry this out in all kind of ways could beweakened.
More research into the impact of the corona virus, split according to the condition of the magnetic presence of the people studied, could be an important step towards a more healthy world.

\section{Conclusion}

With twin physics it is possible to describe four distinct types of electrons, two of them existing inside a finite magnetic space, being the valence- and the conducting electrons. Molecular protons and neutrons are as well accompanied by magnetic spaces. All together a molecule exists inside a magnetic cell, being an addition of these finite magnetic spaces. This opens the possibility to investigate magnetism in an entire new way.

The approach of twin physics, avoiding algebraic calculations and describing results in a geometric way, is relatively easy to apply to specific experimental problems without losing the overview. To facilitate research upon human beings still further, a simplified version without formulas, called the twin model, is created. In this view, all magnetic cells of a human together form a coherent magnetic presence in and around the body. In daily life, this is called the spirit, serving non-biological functions, like religion, music, poetry, painting and other arts.

This suggests that medical and pharmaceutical research not only overlooks the spirit, but that also the possible effects of medication upon spiritually strongly developed people remain beyond observation. Thus for this type of people, medicines might worsen disease, instead of curing.

In a previous paper [7], we explained that the corona virus, because of its form, is expected to have a relatively large region of magnetic influence around it; people having a feeble magnetic presence are expected to be more susceptible for it and will suffer more than those having a strong and healthy magnetic presence. More research into the impact of the corona virus, split according to the condition of the magnetic presence of the people studied, could be an important step towards a more healthy world. Because it is a rather unexplored area, some suggestions are given about how to set up a reasonably reliable study, like comparing groups of musicians or artists with similar groups of people having nonartistic professions, considering the percentage having been in a hospital and how many of them needed intensive case.

All individuals possess a unique magnetic presence and will experience unhealthy periods; possibly artists could help. For that 
reason, striving to a vaccination degree of $100 \%$ could be dangerous for the health of the entire population if this would affect this specific group of people. It could be interesting to compare the percentage of artists and musicians refusing vaccination with a control group, to find out mutual deviations.

If the corona virus is a kind of correcting phenomenon for the complete humanity, and if indeed its magnetic cell connects with the magnetic presence of humans, then it might be dangerous trying to expel it by undifferentiated vaccination, as the next correction could be worse.

\section{Acknowledgements}

I am very grateful to Pieter Dekker for the stimulating discussions and the correction of the scientific content. I want to express my gratitude to Hans Graef for his swift and involved way of correcting the language.

\section{Conflict of Interest}

The author declares no conflict of interest.

\section{Bibliography}

1. Backerra ACM. "Uncertainty as a principle". Physics Essays 23.3 (2010): 419-441.

2. Davisson C and Germer L. "Reflection of electrons by a crystal of Nickel". Proceedings of the National Academy of Sciences of the United States of America 14 (1928): 317-322.

3. Heisenberg W. "Schritte über Grenzen”. Erweiterte Ausgabe. R. Piper and Co. Verlag, München (1971). English translation by Peter Heath: Heisenberg W. (1974). "Schritte über Grenzen". New York, Harper and Row.

4. Jammer M. "The Philosophy of Quantum Mechanics". John Wiley and Sons, New York, NY (1974): 104.

5. Backerra ACM. "Deviating features of protons, neutrons and electrons on a nano scale". Advances in Nanoscience and Nanotechnology 3.1 (2019): 1-9.

6. Backerra ACM. "Four Types of electrons and their Associated Finite Magnetic Fields". Nano Progress 3.5 (2021): 17-33.
7. Backerra ACM. "The importance of magnetism in understanding the impact of the corona virus". Journal of Nanomedicine and Biotherapeutic Discovery 10.2 (2020): 1-9.

8. Backerra ACM. "A shift in theoretical attention for the properties of bulk materials to those of the borders". International Journal of Nanotechnology and Nanomedicine 4.1 (2019): 1-8.

9. Kahn P J. "Introduction to linear algebra”. London: Harper and Row, Ltd. (1967): 17-40.

10. Backerra ACM. "Four Types of Electrons and their Associated Finite Magnetic Fields". Nano Progress 3.5 (2021): 17-33.

11. Backerra A. "Relation between Planck's constant and speed of Light, predicting proton radius more accurately". Applied Physics Research 11.5 (2019): 1-9.

\section{Assets from publication with us}

- Prompt Acknowledgement after receiving the article

- Thorough Double blinded peer review

- Rapid Publication

- Issue of Publication Certificate

- High visibility of your Published work

Website: www.actascientific.com/

Submit Article: www.actascientific.com/submission.php Email us: editor@actascientific.com

Contact us: +919182824667 